\title{
On the role of permanent dipoles in second harmonic generation
}

\author{
David L Andrews $\nmid \S$ and William J Meath $\div$ - \\ $\dagger$ Centre for Interdisciplinary Studies in Chemical Physics, The University of Western Ontario, \\ London, Ontario, Canada N6A 3K7 \\ $\ddagger$ Department of Chemistry, The University of Western Ontario, London, Ontario, Canada \\ N6A 5B7
}

Received 22 July 1993

\begin{abstract}
The significance of permanent electric dipoles in second harmonic generation is considered with regard to their role in both the non-linear susceptibility and also in the orientation of polar molecules. Under conditions of near-resonance with the first electronic excited state, the susceptibility is dominated by a two-level term governed by the difference between the ground- and excited-state dipoles; this term also drives the harmonic conversion in electric field-induced second harmonic generation. It is shown that if the resonant level is not the lowest electronic excited state, there is a secondary resonance feature which can contribute just as strongly as the two-level term, and which can dominate the harmonic conversion if the ground- and excited-state dipoles are either equal, or both zero.
\end{abstract}

\section{Introduction}

It is well known that the coherent generation of even optical harmonics is generally forbidden in isotropic media [1,2]. For this reason, the coherent production of second harmonics in fiuids or disordered solids generally requires a degree of orientational order, unless the intensity of the laser pump is sufficiently high that six-wave interactions come into play [3]. Where polar molecules constitute at least part of the isotropic medium, application of a static electric field provides an obvious and widely used means of conferring the required degree of anisotropy.

Mechanisms for second harmonic generation (SHG) which require a permanent electric dipole moment can only be satisfied by non-centrosymmetric species, which necessarily also possess the required non-zero hyperpolarizability [4]. In the case of fluids [5] the field is applied concurrently with the pump laser, a technique known as electric field-induced second harmonic generation (EFISHG). For disordered solids such as amorphous polymers [6,7], the static field may be applied during solidification from a melt phase, producing a solid whose in-built orientational order permits the observation of SHG even after the field is removed.

The effects of diagonal dipole moment matrix elements, including permanent electric dipoles, on the absorption of radiation through single- and multi-photon laser-molecule interactions have been investigated previously, both for two- and many-level molecules [8-17]. In this paper the significance of permanent electric dipoles in SHG is reconsidered with particular reference to the two-level model widely employed in both linear and non-linear optics [18-23]. In section 2 the general working equations are set out, and in section 3 it is shown how

§ Permanent address: School of Chemical Sciences, University of East Anglia, Norwich NR4 7TJ, UK.

I Associated with the Centre for Interdisciplinary Studies in Chemical Physics, University of Western Ontario, London, Ontario, Canada N6A 3K7. 
contributions associated with the two-level model can be separated from those involving other levels. Specificaliy, it is shown under what conditions the two-level contributions, which involve the difference of the permanent dipoles for the two states, dominate in resonance harmonic generation. Equations for the harmonic intensity under these conditions are derived, and the essential features of the paper are summarized in section 4.

\section{Second harmonic generation-general working equations}

A convenient starting point for a treatment based on time-dependent perturbation theory is the Fermi golden rule equation

$$
\Gamma=\frac{2 \pi}{h}\left|\sum_{\xi} M_{F I}(\xi)\right|^{2} \rho_{\omega}
$$

where $M_{F I}(\xi)$ is the matrix element connecting the initial and final states for the process for a molecule $\xi$ and $\rho_{\omega^{\prime}}$ is the density of final states for the emitted radiation, given by [24]

$$
\rho_{\omega^{\prime}}=\left(8 \pi^{3} \hbar c^{3}\right)^{-1} \omega^{\prime 2} V \mathrm{~d} \Omega
$$

where $V$ is the quantization volume and $\mathrm{d} \Omega$ is an element of solid angle about the propagation direction. Because for each participating molecule the initial and final states in the harmonic conversion process are identical, the matrix element is summed over all such molecules. This leads to quantum interference between terms associated with different molecules; moreover, the interference is only fully constructive for emission in the forward direction. As will become evident, this is the origin of the coherence properties characterizing the laser-like harmonic emission [4]. Time-dependent perturbation theory leads to the following expansion for the matrix element for any one molecule:

$$
\begin{aligned}
M_{F I}=\left\langle F\left|H_{\mathrm{int}}\right| I\right\rangle & +\sum_{R \neq I} \frac{\left\langle F\left|H_{\mathrm{int}}\right| R\right\rangle\left\langle R\left|H_{\mathrm{int}}\right| I\right\rangle}{\left[E_{l}-\left(E_{R} \pm \frac{1}{2} \mathrm{i} \hbar \gamma_{R}\right)\right]} \\
& +\sum_{R \neq I} \sum_{S \neq I} \frac{\left\langle F\left|H_{\mathrm{int}}\right| S\right\rangle\left\langle S\left|H_{\mathrm{int}}\right| R\right\rangle\left\langle R\left|H_{\mathrm{int}}\right| I\right\rangle}{\left[E_{I}-\left(E_{S} \pm \frac{1}{2} \mathrm{i} \hbar \gamma_{S}\right)\right]\left[E_{I}-\left(E_{R} \pm \frac{1}{2} \mathrm{i} \hbar \gamma_{R}\right)\right]}
\end{aligned}
$$

where it is understood that the sums over intermediate states $R, S$ etc are taken over states of the dynamical system comprising both the molecule and the radiation: $I$ and $F$ denote the initial and final system states. The phenomenological inclusion of damping factors in the energy denominators produces the resonant Lorentzian lineshape characteristic of homogeneous linebroadening processes. Each parameter $\gamma$ may be considered a sum of the inverse lifetimes associated with line-broadening, and represents a FWHM linewidth. The sign of each damping factor, $\pm \frac{1}{2} \mathrm{i} h \gamma$, is dictated by the causality condition that all singularities must lie in the lower half of the complex frequency plane [25]. This rule leads to a negative sign in the denominator terms that can exhibit resonance, i.e. those terms whose real parts may vanish when certain molecular-state and photon energies match, and a positive sign in terms which can only exhibit anti-resonance (equivalent to resonance at a negative frequency). The interaction operator $H_{\mathrm{int}}$ in (3) is the electric dipole interaction operator whose quantum electrodynamical expression is as follows [26]:

$$
H_{\text {int }}(\xi)=-\mathrm{i} \sum_{k, \lambda}\left\{\frac{\hbar \omega}{2 \varepsilon_{0} V}\right\}^{1 / 2}\left\{\mu(\xi) \cdot e^{(\lambda)}(k) a^{(\lambda)}(k) \mathrm{e}^{\mathrm{i} k \cdot R_{\xi}}-\mu(\xi) \cdot \bar{e}^{(\lambda)}(k) a^{\dagger(\lambda)}(k) \mathrm{e}^{-\mathrm{i} k \cdot R_{\xi}}\right\} .
$$




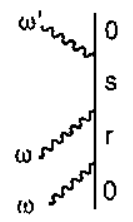

(a)

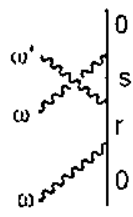

(b)

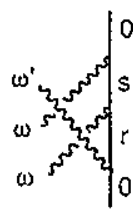

(c)
Figure 1. Time-ordered diagrams for second harmonic generation, time progressing upwards.

Here the sum is over radiation modes with wavevector $k$, circular frequency $\omega=c|k|$ and polarization $e^{(\lambda)}$, each associated with a creation operator $a^{\dagger(\lambda)}(k)$ and an annihilation operator $a^{(\lambda)}(k)$. The vector $\bar{e}^{\prime(\lambda)}$ is the complex conjugate of $e^{\prime(\lambda)}$ : allowing for the polarization vector to be complex accommodates circular as well as plane polarizations. Since each operation of the interaction operator $H_{1 \mathrm{tat}}$ results in either the absorption or the emission of one photon, it is the third-order term in (3) which represents the leading contribution for the three-photon process of SHG. Its explicit form is commonly evaluated by reference to the three time-ordered diagrams shown in figure 1; these represent the possible sequences of radiation intermediate states, and in each case it remains necessary to sum over intermediate states $r$ and $s$ of the molecule (lower case symbols being adopted for the molecular levels). One of the key features to be noted at this stage is that these summations do not exclude the ground or any other state $[8,10,11,27]$. The time-ordered diagrams make it clear why this is so: it is because intermediate states of the system invariably differ from the initial state by virtue of the changes in the radiation field.

For pump laser radiation comprising $n$ photons of wavevector $k$, circular frequency $\omega=c k$ and polarization vector $e$, and an emergent harmonic with $n^{\prime}$ photons of wavevector $k^{\prime}$, frequency $\omega^{\prime}=c k^{\prime}=2 \omega$ and polarization $e^{\prime}$, the result for $M_{F I}(\xi)$ has the following structure [2]:

$$
M_{F I}(\xi)=-\frac{\mathrm{i}}{2}\left\{\frac{\hbar \omega}{\varepsilon_{0} V}\right\}^{3 / 2}\left\{n(n-1)\left(n^{\prime}+1\right)\right\}^{1 / 2} \beta_{i j k}(\xi) \bar{e}_{\mathrm{t}}^{\prime} e_{j} e_{k} \mathrm{e}^{\mathrm{i} \Delta k \cdot R_{\xi}}
$$

where summation over repeated indices is implied. In $(5) \beta_{i j k}(\xi)$ is the second-order susceptibility (hyperpolarizability) tensor for molecule $\xi$ at a position $R_{\xi}$ as defined by [28]

$$
\begin{aligned}
\beta_{i j k}=\sum_{r} \sum_{s} & {\left[\frac{\mu_{i}^{0 s} \mu_{j}^{s r} \mu_{k}^{r 0}}{\left(E_{s 0}-2 \hbar \omega-\frac{1}{2} \mathrm{i} \hbar \gamma_{s}\right)\left(E_{r 0}-\hbar \omega-\frac{1}{2} \mathrm{i} \hbar \gamma_{r}\right)}\right.} \\
& +\frac{\mu_{j}^{0 s} \mu_{i}^{s r} \mu_{k}^{r 0}}{\left(E_{s 0}+\hbar \omega+\frac{1}{2} \mathrm{i} \hbar \gamma_{s}\right)\left(E_{r 0}-\hbar \omega-\frac{1}{2} \mathrm{i} \hbar \gamma_{r}\right)} \\
& \left.+\frac{\mu_{j}^{0 s} \mu_{k}^{s r} \mu_{i}^{r 0}}{\left(E_{s 0}+\hbar \omega+\frac{1}{2} \mathrm{i} \hbar \gamma_{s}\right)\left(E_{r 0}+2 \hbar \omega+\frac{1}{2} \mathrm{i} \hbar \gamma_{r}\right)}\right]
\end{aligned}
$$

and $\Delta k$ is the wavevector mismatch defined by

$$
\Delta k=2 k-k^{\prime}
$$

The result (5) for the molecular matrix element is now inserted into the rate equation (1), using (2) for the density of radiation states, having regard to the need to perform an average over molecular orientations. Assuming that there is no correlation between molecular position and orientation, the sum over molecules in (1) can effectively be separated as a product of an 


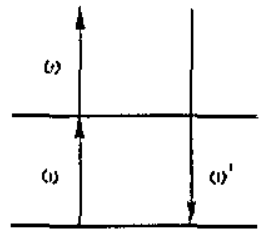

(a)

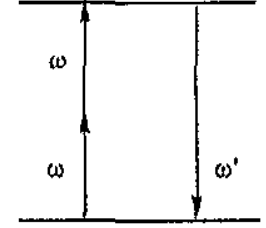

(b)
Figure 2. Energy level diagrams for SHG under conditions of single resonance $(a)$ at the fundamental, and $(b)$ at the harmonic frequency.

orientationally averaged, position-independent, term and a coherence factor $\eta_{N}$ comprising a sum of the position-dependent phase factors

$$
\eta_{N}=\left|\sum_{\xi}^{N} \exp \left(\mathrm{i} \Delta k \cdot R_{\xi}\right)\right|^{2} .
$$

It then follows that the radiant intensity of second harmonic radiation from an interaction region containing $N$ molecules is given by [2]

$$
\mathcal{I}_{\text {SHG }}=\left(\omega^{4} I_{\omega}^{2} g_{\omega}^{(2)} / 2 \pi^{2} c^{5} \varepsilon_{0}^{3}\right) \eta_{N}\left|\left\langle\beta_{i j k} e_{i}^{\prime} e_{j} e_{k}\right\rangle\right|^{2}
$$

where $I_{\omega}$ is the irradiance (power per unit cross-sectional area) of the pump and $g_{\omega}^{(2)}$ its degree of second-order coherence [29]. The angular brackets in (9) denote an orientational average which has to be effected with regard to a temperature-dependent distribution of molecular orientations. By converting the sum over molecules in the coherence factor ( 8 ) to a continuous integral, the familiar $\operatorname{sinc}^{2}\left(\frac{1}{2}|\Delta k| L\right)$ dependence on the propagation distance $L$ is obtained $\left(\operatorname{sinc} x=x^{-1} \sin x\right)$.

\section{The effects of permanent dipoles}

\subsection{Resonance, the two-level model and the significance of other levels}

Under near-resonance conditions, a particular excited state $|u\rangle$ of the material dominates in the sums over intermediate molecular states in (6). The result is an increase in the magnitude of the susceptibility, thus producing an enhanced rate of non-linear optical conversion. However, this is not the only factor which contributes to an increase in the conversion efficiency under near-resonance conditions, since realization of phase-matching $\Delta k \approx 0$ to optimize the degree of coherent output (by maximising the $\operatorname{sinc}^{2}$ function) also commonly necessitates working in regions of anomalous dispersion close to an absorption band [4].

As is evident from the form of the first term in the expression for the second-order susceptibility (6), resonances may occur both at the fundamental and at the harmonic frequency (see figure 2). Such situations arise when the energy of either one incident photon $(\hbar \omega)$ or the sum energy of two incident photons ( $2 \hbar \omega)$ closely matches that of an excited state of the molecule. In principle both conditions may be simultaneously satisfied when two excited states are involved, giving rise to double resonance. In the more usual case of a single resonance (i.e. either as in figure $2(a)$ or $2(b)$, harmonic conversion is largely driven by transitions involving just the ground and resonant levels, so that the kinetics approximate to those of a two-level system. It will not be necessary at this stage to specify which of the two resonance conditions is obtained. It transpires that in the realm of resonant multiphoton phenomena the two-level approximation is peculiarly appropriate for harmonic emission; most processes in which the molecule does not return to its ground state, such as $n$-photon absorption, require three or more levels for their proper representation. 
To begin with, it is useful to separate the susceptibility into a sum of two terms, in the first of which both summations over intermediate states $|r\rangle$ and $|s\rangle$ are restricted to the ground level $|0\rangle$ and a resonant level $|u\rangle$, and in the second term all other possibilities are accounted for [38]. Hence we can write

$$
\beta_{i j k}=\beta_{i j k}^{\mathrm{TLA}}+\beta_{i j k}^{\prime}
$$

where TLA denotes the two-level approximation. It ought to be noted at the outset that the second term in (10) does not completely exclude the states $|0\rangle$ and $|u\rangle$ from its intermediate state summations; for example it accommodates contributions associated with $|r\rangle=\{u\rangle,|s\rangle \neq\{|0\rangle,|u\rangle\}$. Focusing first on the two-level term, there are clearly four contributions to add $(|r\rangle=|0\rangle,|u\rangle ;|s\rangle=|0\rangle,|s\rangle)$ for each of the three susceptibility terms. The sum of the terms with $|r\rangle=|0\rangle,|s\rangle=|0\rangle$ vanishes identically and careful analysis of the other terms, having regard to the proper signs for the damping corrections, yields a result comprising six terms, which may be expressed as follows:

$$
\begin{aligned}
\beta_{u k}^{\mathrm{TLA}} \bar{e}_{i}^{\prime} e_{j} e_{k}= & \frac{\left(\mu^{0 u} \cdot \bar{e}^{\prime}\right)\left(\mu^{0 u} \cdot e\right)(d \cdot e)}{\left(E_{u 0}-2 \hbar \omega-\frac{1}{2} \mathrm{i} \hbar \gamma_{u}\right)\left(E_{u 0}-\hbar \omega-\frac{1}{2} \mathrm{i} \hbar \gamma_{u}\right)} \\
& +\frac{\left(\mu^{0 u} \cdot e\right)^{2}\left(d \cdot \bar{e}^{\prime}\right)}{\left(E_{u 0}+\hbar \omega+\frac{1}{2} \mathrm{i} \hbar \gamma_{u}\right)\left(E_{u 0}-\hbar \omega-\frac{1}{2} \mathrm{i} \hbar \gamma_{u}\right)} \\
& +\frac{\left(\mu^{0 u} \cdot \bar{e}^{\prime}\right)\left(\mu^{0 u} \cdot e\right)(d \cdot e)}{\left(E_{u 0}+\hbar \omega+\frac{1}{2} \mathrm{i} \hbar \gamma_{u}\right)\left(E_{u 0}+2 \hbar \omega+\frac{1}{2} \mathrm{i} \hbar \gamma_{u}\right)}
\end{aligned}
$$

assuming only that the damping is small compared with the harmonic frequency in the numerator of one term, and the electric dipole transition moments are real. The vector parameter $d$ in (11) defined by

$$
d=\mu^{u u}-\mu^{00}
$$

represents the difference between the static electric dipole moments of the resonant and ground states. The first term on the right-hand side of (11) dominates in the case of a two-photon resonance; both first and second terms contribute strongly in the case of a one-photon resonance. For conciseness in later results, it is helpful to write the complete result in the form

$$
\begin{aligned}
\beta_{\mathrm{r} j k}^{\mathrm{TLA}} \bar{e}_{i}^{\prime} e_{j} e_{k}=[ & \left(\mu^{0 u} \cdot \bar{e}^{\prime}\right)\left(\mu^{0 u} \cdot e\right)(d \cdot e)\left\{\left(\delta_{-2} \delta_{-1}\right)^{-1}+\left(\delta_{+1} \delta_{+2}\right)^{-1}\right\} \\
& \left.+\left(\mu^{0 u} \cdot e\right)^{2}\left(d \cdot \bar{e}^{\prime}\right)\left(\delta_{+1} \delta_{-1}\right)^{-1}\right]
\end{aligned}
$$

where $\delta_{-2}, \delta_{-1}, \delta_{+1}$ and $\delta_{+2}$ are the energy denominator factors as defined by

$$
\begin{aligned}
& \delta_{-2}=E_{u 0}-2 \hbar \omega-\frac{1}{2} \mathrm{i} \hbar \gamma_{u} \\
& \delta_{-1}=E_{u 0}-\hbar \omega-\frac{1}{2} \mathrm{i} \hbar \gamma_{u} \\
& \delta_{+1}=E_{u 0}+\hbar \omega+\frac{1}{2} \mathrm{i} \hbar \gamma_{u} \\
& \delta_{+2}=E_{u 0}+2 \hbar \omega+\frac{1}{2} \mathrm{i} \hbar \gamma_{u} .
\end{aligned}
$$

When the effects of damping are ignored and $e \| \vec{e}^{\prime}$, factorization of the scalar products in (13) can be effected and the following simpler result $[30,31]$ applies:

$$
\begin{aligned}
\beta_{i j k}^{\mathrm{TLA}} \bar{e}_{i}^{\prime} e_{j} e_{k} & =\left(\mu^{0 u} \cdot e\right)^{2}(d \cdot e)\left\{\left(\delta_{-2} \delta_{-1}\right)^{-1}+\left(\delta_{+1} \delta_{-1}\right)^{-1}+\left(\delta_{+1} \delta_{+2}\right)^{-\mathrm{I}}\right\} \\
& =3\left(\mu^{0 u} \cdot e\right)^{2}(d \cdot e) E_{u 0}^{2} /\left(E_{u 0}^{2}-4 \hbar^{2} \omega^{2}\right)\left(E_{u 0}^{2}-\hbar^{2} \omega^{2}\right)
\end{aligned}
$$


The most significant feature in both the general and the simplified results (13) and (18) is the appearance in each term of the vector parameter $d$. Clearly it is important to have included the ground state of the molecule in the sums over intermediate states for the dependence on $d$ to be recovered, and this is important for two reasons. First, the result (13) shows that the two-level susceptibility can be supported only by molecules with permanent ground- or excited-state dipoles, which means polar molecules. Second, it is clear that the two-level susceptibility should be considerably enhanced in polar molecules having a large $d$, such as 'giant dipole' molecules displaying charge transfer in their excited states [14, 15, 30-35, 38]. Similar features arise in the theory of multi-photon absorption [8-17,33,34].

Suppose we now take the specific case of a medium possessing an excited state $|u\rangle$ close in energy to that of the emitted harmonic, $2 \hbar \omega$. For practical application this condition is generally more useful than resonance at the fundamental frequency since the latter condition is likely to result in a substantial loss of pump power through decay following conventional single-photon absorption [2]. In view of its denominator structure, it is clearly the first term in (13) which will provide the major two-level contribution to the susceptibility:

$$
\beta_{i j k}^{\mathrm{TLA}} \bar{e}_{i}^{\prime} e_{j} e_{k} \approx \frac{\left(\mu^{0 u} \cdot \bar{e}^{\prime}\right)\left(\mu^{0 u} \cdot e\right)(d \cdot e)}{\hbar^{2}\left(\Delta \omega-\frac{1}{2} \mathrm{i} \gamma_{u}\right)\left(\omega+\Delta \omega-\frac{1}{2} \mathrm{i} \gamma_{u}\right)}
$$

where $\Delta \omega$ represents the detuning from resonance. Both first and second terms of (13) dominate in the case of resonance at the fundamental frequency. If $d$ vanishes, as for example in the case of a tetrahedral molecule, the entire two-level susceptibility disappears. Nonetheless for any such non-centrosymmetric molecule the second term of (10) generally persists and is dominated by the term with essentially the same denominator structure as (19), i.e. the product of a near-resonant and off-resonant term. Under such circumstances the $\beta^{\prime}$ susceptibility may still be conveniently represented as the sum of a resonant and a non-resonant part:

$$
\begin{aligned}
& \beta_{i j k}^{\prime}=\beta_{i j k}^{\text {res }}+\beta_{i j k}^{\text {non-res }} \\
& \beta_{i j k}^{\text {res }}=\frac{\mu_{i}^{0 u}}{\hbar\left(\Delta \omega-\frac{1}{2} \mathrm{i} \gamma_{u}\right)} \sum_{r \neq 0, u} \frac{\mu_{j}^{u r} \mu_{k}^{r 0}}{\left(E_{r 0}-\hbar \omega-\frac{1}{2} \mathrm{i} \hbar \gamma_{r}\right)} \\
& \beta_{i j k}^{\text {oon-res }}=\left(\sum_{r \neq 0, u} \sum_{s \neq u}+\sum_{r=0, u} \sum_{s \neq 0, u}\right) \frac{\mu_{i}^{0 s} \mu_{j}^{s r} \mu_{k}^{r 0}}{\left(E_{s 0}-2 \hbar \omega-\frac{1}{2} \mathrm{i} \hbar \gamma_{s}\right)\left(E_{r 0}-\hbar \omega-\frac{1}{2} \mathrm{i} \hbar \gamma_{r}\right)} \\
& \quad+\left(\sum_{r \neq 0, u} \sum_{s=0, u}+\sum_{r} \sum_{s \neq 0, u}\right)\left[\frac{\mu_{j}^{0 s} \mu_{i}^{s r} \mu_{k}^{r 0}}{\left(E_{s 0}+\hbar \omega+\frac{1}{2} \mathrm{i} \hbar \gamma_{s}\right)\left(E_{r 0}-\hbar \omega-\frac{1}{2} \mathrm{i} \hbar \gamma_{r}\right)}\right. \\
& \left.\quad+\frac{\mu_{j}^{0 s} \mu_{k}^{s r} \mu_{i}^{r 0}}{\left(E_{s 0}+\hbar \omega+\frac{1}{2} \mathrm{i} \hbar \gamma_{s}\right)\left(E_{r 0}+2 \hbar \omega-\frac{1}{2} \mathrm{i} \hbar \gamma_{r}\right)}\right] .
\end{aligned}
$$

When $E_{u 0} \approx 2 \hbar \omega$ and the detuning $\Delta \omega$ is small, the resonant part of the susceptibility strongly dominates over the non-resonant background contribution, and it is evident that the former factorizes into the product of a resonant term associated with the $|0\rangle \rightarrow|u\rangle$ transition and an off-resonant term associated with the $|u\rangle \rightarrow|r\rangle \rightarrow|0\rangle$ transition. It is interesting to consider the behaviour under various conditions. (i) Assuming that the level $u$ is the lowest electronic excited state, comparison of (19) and (21) shows that the two-level susceptibility will normally dominate over the resonant remainder, provided of course that $d \neq 0$. In other words, the harmonic response is largely driven by the difference between the ground- and excited-state permanent dipoles. (ii) If $u$ is not the lowest level then both the two-level susceptibility (19) 
and the resonance feature (21) can contribute strongly, again provided that $d \neq 0$. (iii) Lastly, if $d$ vanishes then the resonant contribution (21) will dominate the harmonic susceptibility.

The above analysis also reveals, that whether or not there is a two-level contribution resulting from a non-zero $d$, the limiting behaviour under resonance conditions is that of a two-stage process entailing two-photon absorption followed by emission of the harmonic. This reflects the fact that if $\Delta \omega^{\prime}$ represents the smallest difference in frequency between any of the molecular energy levels and either the fundamental or the harmonic radiation, the entire threephoton conversion process must be complete within a time $\sim\left(\Delta \omega^{\prime}\right)^{-1}$. The principal difference between frequency doubling under nearly exact resonance and substantially off-resonance conditions thus lies in the fact that in the former an appreciable, and indeed measurable, timelag can exist between the absorption and the emission. This is because the approximate energy conservation in the two-photon absorption stage allows the intermediate state $|u\rangle$ to exist for a time interval which is no longer so severely constrained by the time-energy uncertainty principle [28]. Also, in the two-stage process any molecular motion which occurs between the absorption and emission stages can lead to a partial loss of coherence.

\subsection{Harmonic intensity in a partially ordered system}

To obtain the explicit result for the harmonic intensity requires evaluation of the ensemble orientational average in (9). Under equilibrium conditions this incorporates the Boltzmann weighting term (exp $\left.\mu^{00} \cdot D / \varepsilon_{0} k T\right)$, where $D$ is the local electric displacement produced by an applied static field and $\mu^{00}$ is the permanent electric dipole moment of the molecule in its ground state. Hence we have

$\overline{\mathcal{I}}_{\mathrm{SHG}}=\left(\omega^{4} I_{\omega}^{2} g_{\omega}^{(2)} / 2 \pi^{2} c^{5} \varepsilon_{0}^{3}\right) \eta_{N}\left|\left\langle\beta_{i j k} \bar{e}_{i}^{\prime} e_{j} e_{k} \exp \left(\mu^{00} \cdot D / \varepsilon_{0} k T\right)\right\rangle\left\langle\exp \left(\mu^{00} \cdot D / \varepsilon_{0} k T\right)\right\rangle^{-1}\right|^{2}$

Evaluating the weighted rotational averages in (23) entails complex tensor calculus [36]. The orienting effect of the electric field is manifest through the appearance of reduced spherical Bessel functions $j_{n}^{\prime}(\gamma)=j_{n}(\gamma) / j_{0}(\gamma), n=1-3$, as given by

$$
\begin{aligned}
& j_{1}^{\prime}(\gamma)=-\mathrm{i}\left[\gamma^{-1}-\operatorname{coth} \gamma\right] \\
& j_{2}^{\prime}(\gamma)=-1-3 \gamma^{-2}+3 \gamma^{-1} \operatorname{coth} \gamma \\
& j_{3}^{\prime}(\gamma)=\mathrm{i}\left[\left(1+15 \gamma^{-2}\right) \operatorname{coth} \gamma-6 \gamma^{-1}-15 \gamma^{-3}\right]
\end{aligned}
$$

whose imaginary argument has a modulus determined by the Boltzmann factor

$$
\gamma=\left|\mu^{00}\right||D| / \varepsilon_{0} k T .
$$

Full details of the result based on application of the general result (6) for the susceptibility are given elsewhere [37]. For the case of interest, where the response is largely driven by the full two-level second-order susceptibility as given by (13), the result for the harmonic intensity takes the following form:

$$
\begin{gathered}
\overline{\mathcal{I}}_{\mathrm{SHG}}=\left(\omega^{4} I_{\omega}^{2} g_{\omega}^{(2)} / 200 \pi^{2} c^{5} \varepsilon_{0}^{3}\right) \eta_{N} \mid 2 i j_{1}^{\prime}(\gamma)\left[\left(3 \beta_{2}-\beta_{1}\right) p_{1}+\left(2 \beta_{1}-\beta_{2}\right) p_{2}\right]-10 j_{2}^{\prime}(\gamma) \beta_{3} p_{4} \\
-\left.i j_{3}^{\prime}(\gamma)\left[\left(5 \beta_{4}-2 \beta_{2}-\beta_{1}\right)\left(5 p_{3}-2 p_{1}-p_{2}\right)\right]\right|^{2}
\end{gathered}
$$

where $p_{1}-p_{4}$ are polarization parameters defined by

$$
\begin{aligned}
& p_{1}=(\hat{D} \cdot e)\left(\bar{e}^{\prime} \cdot e\right) \\
& p_{2}=\left(\hat{D} \cdot \bar{e}^{\prime}\right)(e \cdot e) \\
& p_{3}=\left(\hat{D} \cdot \bar{e}^{\prime}\right)(\hat{D} \cdot e)^{2} \\
& p_{4}=(\hat{D} \cdot e)\left(\bar{e}^{\prime} \times e\right) \cdot \hat{D}
\end{aligned}
$$


(note that $(e \cdot e)$ is unity only for plane-polarized radiation), and the explicit form taken by the molecular parameters $\beta_{1}-\beta_{4}$ in the two-level case is as follows:

$$
\begin{aligned}
& \beta_{1}=\left[\left(\mu^{u 0} \cdot \mu^{00}\right)\left(\mu^{u 0} \cdot d\right)\left\{\left(\delta_{-2} \delta_{-1}\right)^{-1}+\left(\delta_{+1} \delta_{+2}\right)^{-1}\right\}+\left(\mu^{00} \cdot d\right)\left|\mu^{u 0}\right|^{2}\left(\delta_{+1} \delta_{-1}\right)^{-1}\right] \\
& \beta_{2}=\frac{1}{2}\left[\left.\left\{\left(\mu^{00} \cdot d\right)\right] \mu^{\mu 0}\right|^{2}+\left(\mu^{\mu 0} \cdot \mu^{00}\right)\left(\mu^{\mu 0} \cdot d\right)\right\}\left\{\left(\delta_{-2} \delta_{-1}\right)^{-1}+\left(\delta_{+1} \delta_{+2}\right)^{-1}\right\} \\
& \left.+2\left(\mu^{\mu 0} \cdot d\right)\left(\mu^{\mu 0} \cdot \mu^{\infty}\right)\left(\delta_{+1} \delta_{-1}\right)^{-1}\right] \\
& \beta_{3}=\frac{1}{2}\left[\left\{\left(\mu^{\mu 0} \times d\right) \cdot \mu^{00}\right\}\left(\mu^{\mu 0} \cdot \mu^{00}\right)\left\{\left(\delta_{-2} \delta_{-1}\right)^{-1}-2\left(\delta_{+1} \delta_{-1}\right)^{-1}+\left(\delta_{+1} \delta_{+2}\right)^{-1}\right\}\right] \\
& \beta_{4}=\left[\left(\mu^{\mu 0} \cdot \mu^{00}\right)^{2}\left(\mu^{00} \cdot d\right)\left\{\left(\delta_{-2} \delta_{-1}\right)^{-1}+\left(\delta_{+1} \delta_{-1}\right)^{-1}+\left(\delta_{+1} \delta_{+2}\right)^{-1}\right\}\right] \text {. }
\end{aligned}
$$

Under commonly applied static field strengths it is the $j_{i}^{\prime}$ term which dominates the result (28); in the low-field limit the Taylor series expansion of the reduced spherical Bessel function $j_{n}^{\prime}(\gamma)$ has a leading term in $\gamma^{n}$ and, for example, a permanent dipole of ID even in a relatively high local field $D \sim 10^{8} \mathrm{~V} \mathrm{~m}^{-1}$ still produces a value for $\gamma$ at room temperature of only $\sim 0.1$. Under most conditions it therefore also suffices to take the low-field limit $j_{1}^{\prime}(\gamma) \approx-\mathrm{i} \gamma / 3$, giving the following simpler result for the emitted harmonic intensity under arbitrary polarization conditions:

$$
\begin{aligned}
\tilde{\mathcal{I}}_{\mathrm{SHG}}=\left(\omega^{4} I_{\omega}^{2} g_{\omega}^{(2)} / 450 \pi^{2} c^{5} \varepsilon_{0}^{5}\right)\left(\left|\mu^{00} \| D\right| / k T\right)^{2} \eta_{N} \\
\\
\times\left|\left(3 \beta_{2}-\beta_{1}\right)(\hat{D} \cdot e)\left(\bar{e}^{t} \cdot e\right)+\left(2 \beta_{1}-\beta_{2}\right)\left(\hat{D} \cdot \bar{e}^{\prime}\right)(e \cdot e)\right|^{2} .
\end{aligned}
$$

It is useful to present the explicit forms of the two-level susceptibility combinations $\left(3 \beta_{2}-\beta_{1}\right)$ and $\left(2 \beta_{1}-\beta_{2}\right)$ which characterize the response

$$
\begin{aligned}
\left(3 \beta_{2}-\beta_{1}\right)=\frac{1}{2}\left[\left(\mu^{00} \cdot d\right)\left|\mu^{u 0}\right|^{2}\left\{3\left(\delta_{-2} \delta_{-1}\right)^{-1}-2\left(\delta_{+1} \delta_{-1}\right)^{-1}+3\left(\delta_{+1} \delta_{+2}\right)^{-1}\right\}\right. \\
\left.+\left(\mu^{\mu 0} \cdot \mu^{00}\right)\left(\mu^{u 0} \cdot d\right)\left\{\left(\delta_{-2} \delta_{-1}\right)^{-1}+6\left(\delta_{+1} \delta_{-1}\right)^{-1}+\left(\delta_{+1} \delta_{+2}\right)^{-1}\right\}\right] \\
\left(2 \beta_{1}-\beta_{2}\right)=\frac{1}{2}\left[\left\{\left(\mu^{00} \cdot d\right)\left|\mu^{u 0}\right|^{2}\left\{-\left(\delta_{-2} \delta_{-1}\right)^{-1}+4\left(\delta_{+1} \delta_{-1}\right)^{-1}-\left(\delta_{+1} \delta_{+2}\right)^{-1}\right\}\right.\right. \\
\left.+\left(\mu^{u 0} \cdot \mu^{00}\right)\left(\mu^{u 0} \cdot d\right)\left\{3\left(\delta_{-2} \delta_{-1}\right)^{-1}-2\left(\delta_{+1} \delta_{-1}\right)^{-1}+3\left(\delta_{+1} \delta_{+2}\right)^{-1}\right\}\right] .
\end{aligned}
$$

In the main, it is evident that the energy denominator combinations are not the same as in (18) even with the neglect of damping, so that the form of result quoted previously in the literature $[30,31]$ for the two-level susceptibility does not always apply. However, in the special case where $e \| \bar{e}^{\prime}$, i.e. the harmonic is resolved for the same polarization component as the pump beam, the polarization factors in (37) add; this gives an overall susceptibility dependence governed by the factor $\left(\beta_{1}+2 \beta_{2}\right)$, leading to the more familiar result when the effects of damping are ignored.

\section{Conclusion}

The effects of permanent dipoles in connection with SHG have been considered with regard to their role both in the non-linear susceptibility and also in the orientation of polar molecules. Under conditions of near-resonance with the lowest electronic excited state, the susceptibility is dominated by a two-level term governed by the difference between the ground- and excited-state dipoles. This term also drives the harmonic conversion in EFISHG. If the resonance level is not the lowest electronic excited state, there is a secondary resonance feature which can contribute just as strongly as the two-level term, and which will itself dominate the harmonic conversion in the rare cases where the ground and the excited state dipoles are either equal, or both zero. 


\section{Acknowledgments}

One of us (DLA) gratefully acknowledges the award of a Senior Visiting Fellowship by the Centre for Chemical Physics at the University of Western Ontario, during the tenure of which this collaborative work was carried out.

\section{References}

[1] Andrews D L 1980 J. Phys. B: At. Mol. Phys. 134091

[2] Andrews D L 1993 Adv. Chem Phys. 85 in press

[3] Andrews D L 1993 Nonlinear Optics to be published

[4] Boyd R W 1992 Nonlinear Optics (London: Academic)

[5] Hauchecorne G, Kerhervé F and Mayer G $1971 \mathrm{~J}$. Physique 3247

[6] Singer K D, Sohn J E and Lalama S J 1986 Appl. Phys. Lett. 49248

[7] Singer K. D, Kuzyk M G and Sohn J E 1987 J. Opt. Soc. Am. B 4968

[8] Dick B and Hohlneicher G 1982 J. Chem. Phys. 765755

[9] Meath W J and Power E A 1984 Mol. Phys. 51585

[10] Meath W J and Power E A 1984 J. Phys. B: At. Mol. Phys. 17763

[11] Meath W J and Power E A 1987 J. Phys. B: At. Mol. Opt. Phys. 201945

[12] Kmetic M A and Meath W J 1985 Phys, Lett. 108A 340

[13] Thuraisingham R A and Meath W J I985 Mol. Phys. 56193

[14] Hattori T and Kobayashi T 1987 Phys. Rev. A 352733

[15] Kondo A. E and Meath W J 1991 Mol. Phys. 74113

[16] Nakai S and Meath W J 1992 J. Chem. Phys. 964991

[17] Kondo A E, Blokker V M and Meath W J 1992 J. Chem. Phys. 962544 and references therein

[18] Rabi II 1937 Phys. Rev. 51652

[19] Bloembergen N and Shen Y R 1964 Phys. Rev. 133 A37

[20] Shirley J H 1965 Phys. Rev. B 138979

[21] Sargent M III, Scully M O and Lamb W E 1974 Laser Physics (New York: Addison-Wesley) ch 2

[22] Allen L and Eberly J H 1975 Optical Resonance and Two-level Asoms (New York: Wiley)

[23] Dion D R and Hirschfelder J O 1976 Adv. Chem. Phys. 35265

[24] Power E A 1964 Introductory Quantum Electrodynamics (London: Longmans)

[25] Butcher P N, Loudon R and McLean T P 1965 Proc. Phys. Soc. 85565

[26] Craig D P and Thirunamachandran T 1984 Molecular Quantum Electrodynamics (London: Academic)

[27] Pantell R H and Puthoff H E 1969 Fundamentals of Quantum Electronics (New York: Wiley) section 5.2

[28] Butcher P N and Cotter D 1990 The Elements of Nonlinear Optics (Cambridge: Cambridge University Press)

[29] Loudon R 1983 The Quantum Theory of Light (Oxford: Clarendon) 2nd edn

[30] Oudar J L 1977 J. Chem. Phys. 67446

[31] Oudar J L and Chemia D S 1977 J. Chem. Phys. 662664

[32] Chemla D S and Zyss J (eds) 1987 Nonlinear Optical Properties of Organic Molecules and Crystals (New York: Academic)

[33] Terauchi M and Kobayashi T 1987 Chem. Phys. Lett. 137, 319

[34] Kmetic M A and Meath W J 1990 Phys. Rev. A 411556

[35] Nilar S H, Thakkar A J and Kondon A E 1993 Can. J. Chem. in press

[36] Andrews D L and Harlow M J 1984 Phys. Rev. A 292796

[37] Andrews D L and Sherborne B S 1986 J. Phys. B. At. Mol. Phys. 194265

[38] Wortmann R, Krämer P. Glania C. Lebus S and Detzer N 1993 Chem. Phys. 17399 